\title{
COMMON APPROACH TO GEOPROCESSING OF UAV DATA ACROSS APPLICATION DOMAINS
}

\author{
G. S. Percivall ${ }^{\text {a, }}$, M. Reichardt ${ }^{\text {a }}$, Trevor Taylor ${ }^{\text {a }}$ \\ ${ }^{a}$ Open Geospatial Consortium, Wayland MA, USA - (gpercivall, mreichardt, ttaylor)@opengeospatial.org
}

KEY WORDS: Geoprocessing, Open Standards, OGC, UAV, UAS

\begin{abstract}
:
UAVs are a disruptive technology bringing new geographic data and information to many application domains. UASs are similar to other geographic imagery systems so existing frameworks are applicable. But the diversity of UAVs as platforms along with the diversity of available sensors are presenting challenges in the processing and creation of geospatial products. Efficient processing and dissemination of the data is achieved using software and systems that implement open standards. The challenges identified point to the need for use of existing standards and extending standards. Results from the use of the OGC Sensor Web Enablement set of standards are presented. Next steps in the progress of UAVs and UASs may follow the path of open data, open source and open
\end{abstract} standards.

\section{GROWTH OF UAV/UAS AS BIG DATA}

The age of Unmanned Aerial Systems (UAVs) also called drones as part of Unmanned Aerial Systems (UASs) has arrived and will grow dramatically over the next few years. Kleiner Perkins Caufield \& Byers" "2015 Internet Trends Report" identifies commercial drone shipments as rising rapidly; the use of drones has very broad application; where drone use was previously held back by regulation that is now changing; so that drones will drive a new wave of big data collection (Meeker). With a recent change in FAA approval process, Commercial use of drones in the US skyrocketing (Popper).

Success will require advancement in all elements of UASs. In particular the rapid evolution of software (image processing, statistical analysis, data fusion, etc.) and the highquality of commercial cameras will define the remote sensing done with UAVs (Salamí). Expensive sensors, such as thermal cameras, LiDAR, radar or multi/hyper-spectrometers will be used only in industrial and research applications. Exponential growth in UAS applications can be anticipated with cheaper sensors. Coupled with online data processing this trend will enable widespread commodity development. Civilians, filmmakers, and many innovative users will want more camerawielding drones (Bonnington). The first international contest for aerial photos by drones was conducted in 2014 in partnership with National Geographic France and the social network Dronestagram (Vrignaud).

UAV technology is developing rapidly from research to applications with the need to identify best practices and standards that enable reuse and quality information for applications.

Defining a common approach based on open standards for processing, exploiting, and disseminating are key to achieving the promise of UAVs. The approach presented here is based on open standards from the Open Geospatial Consortium (OGC Standards) and other international standard developing organizations.

\section{FRAMEWORKS FOR UAS}

UASs are similar to other geographic imagery systems. The UAS Production Process at USGS has defined a basic framework (Figure 2) (USGS) that is similar to imagery approaches defined by in ISO Standards (ISO 19101-2) and the US Department of Defense (DoD) for other geographic observation system. The DoD uses the acronym TCPED to define a process similar to Figure 2: Tasking aircraft, Collecting data, Processing that data, Exploiting the data into useful products, and Disseminating the data and products

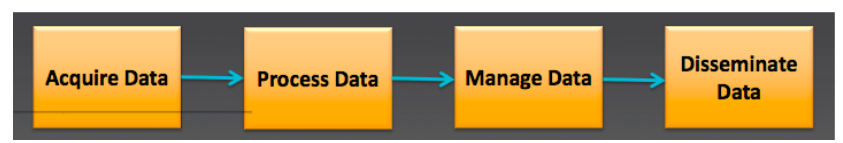

Figure 1. UAS Production Process (USGS)

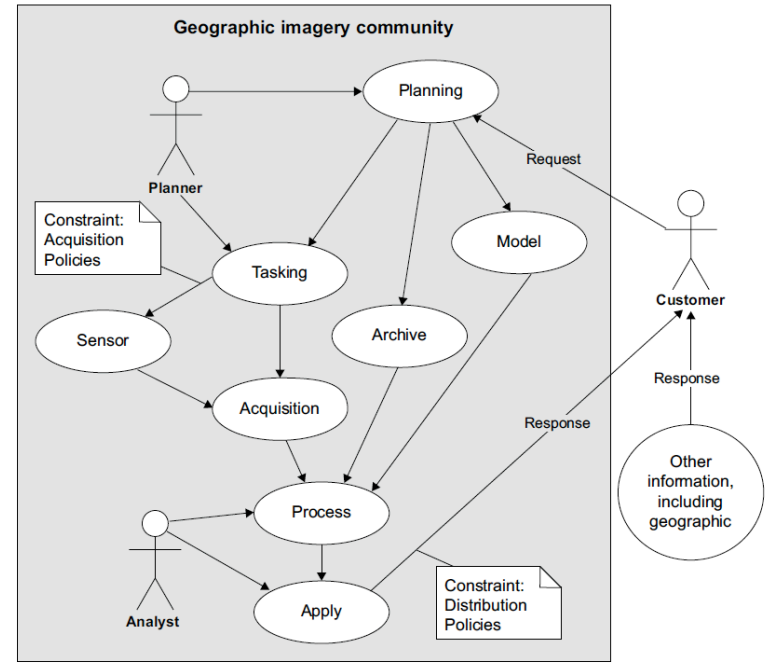

Figure 2. Geospatial Imagery Observation Request Process (ISO 19101-2)

* Corresponding author 
A framework developed from a research point of view is shown in (Figure 3) (Ma). At the data acquisition level, flight, autonomy, and trajectory are addressed. Data processing included photography, image matching, and mosaicking and classification and finally, the applications, were categorized as: environment and agriculture, terrain extraction, 3D visualization and monitoring of hazards.

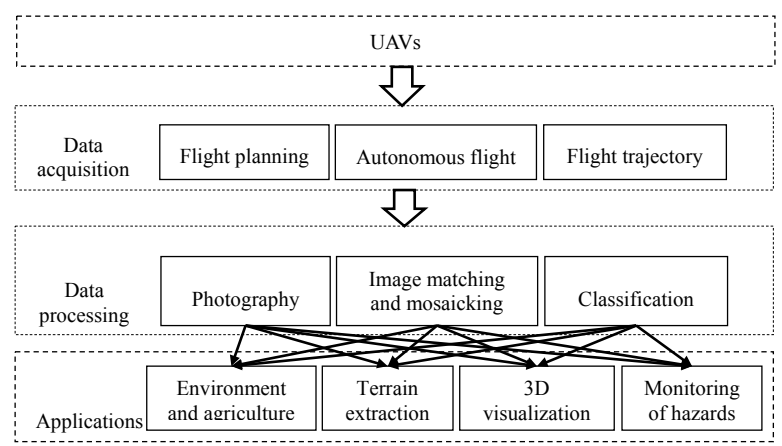

Figure 3. Framework for research on UAVs (Ma)

(Colomina) provide an extensive review related to data processing techniques considering that, in the context of photogrammetry from UAVs, their performance is similar to products from piloted, airborne-based systems:

(a) Image orientation for navigation and camera calibration to cope with the problem of irregularity of frames acquired from UAVs, where computer vision techniques provide some solutions, such as SfM or automatic tie point generation based on point detection and descriptors with sufficient accuracies like SIFT, their variants and many others; and

(b) Surface reconstruction, to obtain DSM and orthophotos with sufficient accuracies in point cloud generation and densification, including multi-view stereopsis techniques.

\section{CHALLENGES WITH UAS}

While the basic UAS frameworks are similar to previous geographic observing systems, significant distinct challenges emerge with UAS technology. To compensate for the limited performance of small, lightweight platforms and acquisition systems more sophisticated processing is required (Colomina and Molina (2014)). Data storage and processing power needed to produce the geospatial products can be substantial (USGS). For example, current cameras capture still images with a 12megapixel sensor that produces a compressed .jpg output file of approximately 1.5 megabytes $(\mathrm{Mb})$. The full-motion video is typically shot at 1,080 pixels with 30 frames per second, which translates to roughly $100 \mathrm{Mb}$ per minute of compressed MP4 video, and although flight durations can vary, most will exceed 30 minutes. As the technology of small, lightweight sensors advances, larger "small" sensors will be available that can produce as much as or even more than $15 \mathrm{Mb}$ of raw, uncompressed data.

There are a number of challenges associated with processing of the UAV imagery. Raw image data, once acquired, must be orthorectified to map standards before it can be used with image data from other platforms or to produce geospatial image products. Each sensor may have unique requirements for preprocessing. Atmospheric correction models and high-quality camera calibration are required. (USGS) (Ma) identifies several processing challenges: Image distortion associated with inexpensive consumer grade digital camera; Difficulty of detecting ground control points gathered from coarser resolution imagery; Limited accuracy of the exterior information (X,Y,Z, roll, pitch, heading).

The most time-consuming steps are image rectification and mosaicking. Both the photogrammetric and the image-matching approaches show promise, but more research and algorithm improvements are needed to provide a smoother, faster workflow from raw to classified imagery (Ma)

As summarized by (Pajares) the quality of photogrammetric products, such as orthophotos, is not always achieved successfully because of the movement of the UAV or due to overlapping errors that require special treatments.

Small UASs are ubiquitous now and are often being tasked to perform traditional mapping production work. Unfortunately, many sensors have low or no metadata which may hinder use and /or reuse of sensor data (BAE).

Legal issues can be specific to UASs. Requirements for accountability and transparency of UAV imagery (Pomfret) may increase requirements on provenance in data processing.

\section{CHALLENGES MOTIVATE THE USE OF STANDARDS}

As the UAS industry develops, the challenges listed in the previous section will be addressed through technology innovations and the development of standards. Standards for geographic observations are quite mature and UASs follow many of those standards already. To advance further, the UASs will need to increase use of existing standards and in some cases new standards will need to be developed.

UAVs have the capability to complement, or even substitute, for satellites and full-scale aircraft. On the negative side, the extremely diverse alternatives of the experiments show a lack of standardization at all levels, from sensors, vegetation indices, operational methods, UAV sizes and models, and automation of pilot capabilities (Salamí).

Government agencies have identified the need for the application of standards. The US Office of the Secretary of Defense (OSD) plans to ensure compliance with the existing DoD/Intelligence Community Motion Imagery Standards Board metadata standard and profiles for all full motion video capable UAS (DoD). The NASA approach for Earth Observations and the Role of UAVs focuses on standards to decrease nonrecurring costs by developing, documenting, and implementing interface standards to support open architecture technology, or "plug and play" (Yuhas).

Standards developing organizations are responding to the need for UAS standards. Cortney Robinson, Secretary of ISO/TC 20/SC 16 on unmanned aircraft systems identifies that standardization in the field of UAS is a timely issue, due to the increasing market demand for civil unmanned aviation vehicles. International Standards are critical in creating the global commercial market (ISO).

In summary, as UAVs become commodity this will drive the increased requirement for plug-and-play capability between the sensor and the platform, between the sensor data output and the downstream processing. To support this trend, standards that 
allow for self-configuration of software are needed. Availability and proliferation of UAV sourced information will require the application of standards to effectively support the proliferation of sensors.

\section{STANDARDS-BASED UAS}

The previous sections identified several challenges associated with UASs as well as how those challenges motivate the need for standards. This section shows the application of OGC standards to UAS with a focus on the Sensor Web Enablement (SWE) standards. All OGC standards including SWE are freely available on the web (OGC-S).

A sensor network is a computer accessible network of many spatially distributed devices using sensors to monitor conditions at different locations. A Sensor Web refers to web accessible sensor networks and archived sensor data that can be discovered and accessed using standard protocols and interfaces. The OGC Sensor Web Enablement (SWE) set of standards enable all types of sensors, instruments, and imaging devices to be accessible and, where applicable, controlled via the Web. SWE standards provide the foundation for "plug-and-play" Webbased sensor networks (Botts).

The SWE Standards are (OGC Standards):

- Observations \& Measurements Schema (O\&M) -Models and XML Schema for encoding observations and measurements from sensors.

- Sensor Model Language (SensorML) - Models and XML Schema for describing sensors systems and processes.

- Sensor Observations Service (SOS) - Web service interface for requesting observations and sensor system information.

- Sensor Planning Service (SPS) - Web service interface for requesting user-driven acquisitions and observations.

- Sensor Alert Service (SAS) - Web service interface for publishing and subscribing to alerts from sensors.

- Web Notification Services (WNS) - Standard web service interface for asynchronous delivery of messages or alerts from SAS and SPS web services and other elements of service workflows.

The SWE standards provide significant benefits for supporting the integration and fusion of a variety of sensors, and readily enables a system that is able to sense and react to threats or opportunities. The SWE standards are in version 2 and implemented up to the highest Technology Maturity Level (Percivall). The SWE standards have been applied to several UAS.

In 2008, on-demand processing of Tigershark UAV full motion video was accomplished using SWE during an US DoD Empire Challenge exercise. The video and UAV navigation information (latitude, longitude, altitude, true heading, pitch, and roll) were served from an SOS. Geolocation of footprints and video were accomplished on-the-fly in a visualization client using SensorML. The video and navigation data from the SOS as well as a sensor model for the video camera, geolocated the video data to the 3D terrain using SensorML (EC08).

As of 2010, NASA and the US Forest Service had flown UAS sensor missions over 57 fires in the western US. The missions demonstrated the capabilities of the Ikhana UAV with a multispectral sensor to provide fire intelligence to management teams (Sullivan). Web access to geospatial processing services for real-time wildfire-derived data products (Figure 4) were implemented utilizing OGC open standards (OGC-S) including:

- Sensor planning service (SPS),

- Web notification service (WNS),

- Web map service (WMS),

- Web coverage service (WCS),

- Web feature service (WFS), and

- KML.

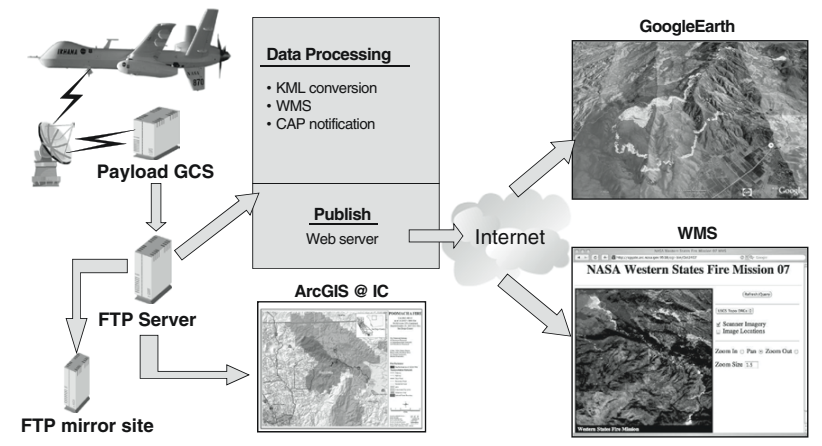

Figure 4. Data collection and distribution of sensor data by Web Services for Ikhana fire observations UAS (Ambrosia)

UAVs equipped with multiple sensors publish data in unpredictable manner.. Additionally, the available approaches only provide proprietary access to the data. Integrating sensors from UAVs into a synchronized data stream is required for publishing the transmitted data on the Web. (Reike) describes a software framework, which simplifies such integration in an interoperable way using OGC SWE standards for a real-world scenario exploring the surface-near atmosphere of the earth.

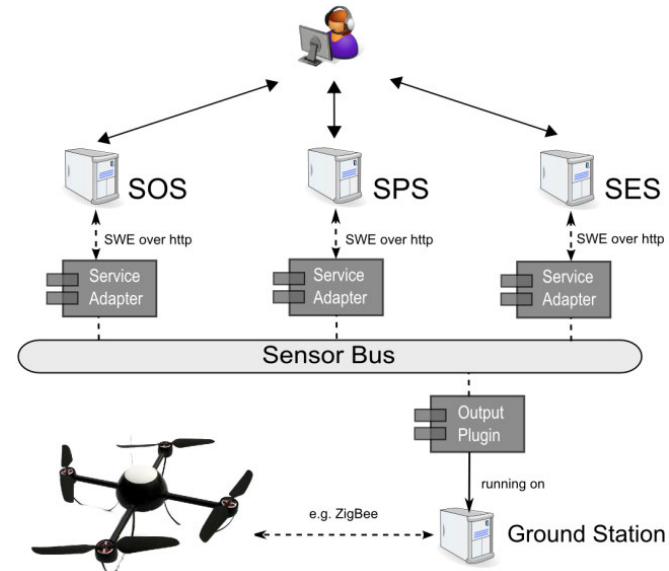

Figure 5. Framework for UAS using OGC SWE (Reike)

Recent research by (Avci) identifies the use of SenorML for the proliferation of sensors on UAVs. As part of mission planning, after the most appropriate UAV is determined, it is time to choose which kind of sensor will be accompany to the UAV. There can be confusion as the number of sensor types and UAV is increasing. Using SensorML it is possible to classify several UAVs (helicopter, quadcopter, blimp and airplane) and cameras (micro analog, HD camera, lowlight and thermal camera) datasheet specifications as a database in XML format.

Combining sensors hosted on UAVs with other sensors in a single framework provide exceptional understanding for given applications. The SWE framework provides for a uniform set of interfaces across a diversity of sensors. (Geipel) uses SWE 
as an infrastructure for precision farming. The use of sensor technologies is standard practice in the domain of precision farming. The variety of vendor-specific sensor systems, control units and processing software has led to increasing efforts in establishing interoperable sensor networks and standardized sensor data infrastructures. The SWE based infrastructure covers the control of sensor systems, the access to sensor data, the transmission of sensor data to web services and the standardized storage of sensor data in a sensor web-enabled server. It permits end users and computer systems to access the sensor data in a well-defined way and to build applications on top of the sensor web services. The infrastructure is scalable to large scenarios, where a multitude of sensor systems and sensor web services are involved. A real-world field trial was set-up to prove the applicability of the infrastructure.

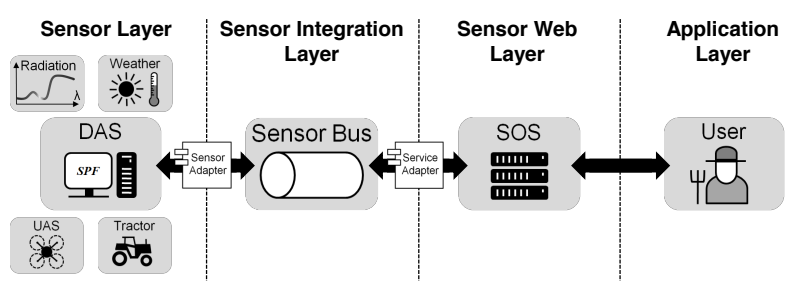

Figure 6. SWE infrastructure for precision farming (Geipel)

The OGC standards have been used successfully for UAS missions. The preceding examples in this section have focused on OGC SWE. Several other OGC standards are also relevant to UASs. Previously mentioned in this section were examples of using WMS, WCS, WFS, and KML (Ambrosia). A full picture of standards relevant to UAS development is provided in Figure 7.

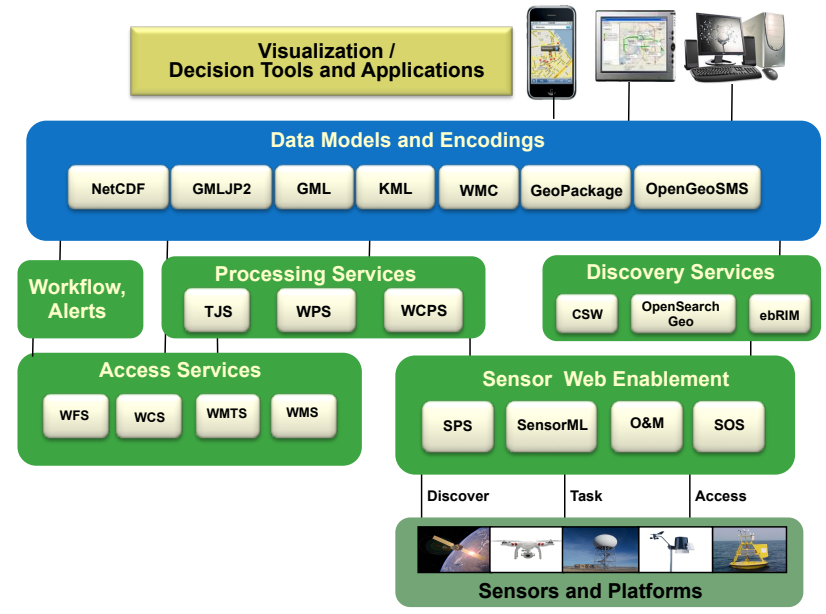

Figure 7. OGC Standards Relevant to UAS

\section{DISCUSSION AND NEXT STEPS}

Open standards provide alternatives to vendor-controlled vertical integration of data collection, database management, analysis, portrayal and user interface. Once a radical idea, componentization is now reality. Small vendors, large vendors, solution providers and integrators can pick and choose components that are likely to "play well together" because of open standards. Small vendors can create components that make them "third party developers" to not one but several of the large vendors.

To gain the full benefit of the explosion of UAV platforms and sensors, the components in UAS will become commodities that will be interchangeable based on open standards. The recent history of software development, Internet connectivity and high data availability has been dominated by the triumvirate approach of Open source software, Open data licenses and Open standards for interfaces and encodings. It appears that UAS development is prepared for similar explosion.

Geographic information derived from UAV hosted sensors is a bringing new insight across all application domains. UAVs introduce many new opportunities to meet user needs. Efficient processing and dissemination of the data based is achieved using software and systems that implement open standards. 


\section{REFERENCES}

Ambrosia, G., Sullivan, D., Buechel, S., Integrating sensor data and geospatial tools to enhance real-time disaster management capabilities: Wildfire observations, Geologic Society of America Monograph (Societal Challenges and Geoinformatics), Chapter 1, GSA Special Paper 482, 2011.

Avci, C., Unmanned Aerial Vehicles Sensing Missions Specification using SensorML, Bachelor's Thesis in Computer Engineering Halmstad University, Technical Report, February 2012.

Bonnington, C., An App-Controlled Drone that Delivers Beautiful 1080P Footage, Wired Gear, 12 May 2014, http://www.wired.com/2014/05/parrot-bebopdrone/?mbid=social_twitter Accessed on 27 July 2015.

Botts, M. Percivall, G., Reed, R., Davidson, J. Sensor Web Enablement: Overview and High Level Architecture, OGC White Paper, OGC document 07-165r1, 2013-04-02.

Colomina, I., P. Molina, P., Unmanned aerial systems for photogrammetry and remote sensing: A review, ISPRS Journal of Photogrammetry and Remote Sensing 92 (2014) 79-97.

DoD, DoD Unmanned Aircraft Systems Roadmap 2005-2030, 4 August 2005.

EC08. Reference available on request from the corresponding author.

Geipel, J. et al., A Sensor Web-Enabled Infrastructure for Precision Farming, ISPRS Int. J. Geo-Inf. 2015, 4, 385-399; doi:10.3390/ijgi4010385

ISO 19101-2, ISO/TS 19101-2:2008, International Standard Geographic information - Reference model - Part 2: Imagery http://www.iso.org/iso/catalogue_detail.htm?csnumber=39983 Accessed on 27 July 2015.

ISO, Gasiorowski-Denis, E., How standards will target the drone industry, ISO News, 27 March 2015 http://www.iso.org/iso/home/news_index/news_archive/news.ht m?refid=Ref1946 Accessed on 27 July 2015.
Ma, L., M. Li, L. Tong, Y. Wang, and L. Cheng, 2013. Using unmanned aerial vehicle for remote sensing application, Proceedings of the IEEE 21st International Conference on Geoinformatics (GEOINFORMATICS), 20-22 June, Kaifeng, China, pp. 1-5.

Meeker, M., 2015 Internet Trends Report, 27 May 2015 http://www.slideshare.net/kleinerperkins/internet-trends-v1 Accessed on 27 July 2015.

OGC Standards. Web page retrieved on 27 July 2015. http://www.opengeospatial.org/standards

Percivall, G., OGC SWE Implementation Maturity Engineering Report, OGC Document 13-032, 2013-09-11.

Popper, B., These are the first 500 companies allowed to fly drones over the US: Welcome to the next generation of aviation, The Verge, 7 July 2015 http://www.theverge.com/2015/7/7/8883821/drone-searchengine-faa-approved-commercial-333-exemptions Accessed on 27 July 2015.

Rieke, M., Foerster, T., Broering, A., Unmanned Aerial Vehicles as MobileMulti-sensor Platforms, The 14th AGILE International Conference on Geographic Information Science; $01 / 2011$.

Salamí, E., Barrado, C. and Pastor, E., UAV Flight Experiments Applied to the Remote Sensing of Vegetated Areas, Remote Sens. 2014, 6, 11051-11081; doi:10.3390/rs61111051

USGS, UAS Future of Land Imaging Presentation to the USGS Executive Leadership Team

Vrignaud, J-P., The First International Contest for Aerial Photos by Drones, National Geographic, 15 May 2014. http://voices.nationalgeographic.com/2014/05/15/the-firstinternational-contest-for-aerial-photos-by-drones/ Accessed on 27 July 2015

Yuhas, C., Earth Observations and the Role of UAVs: A Capabilities Assessment, NASA, Version 1.1, August 2006. 